\title{
NON-LINEAR OPTICS STUDIES AT THE ESRF
}

\author{
A. Ropert, L. Farvacque, ESRF, BP 220, 38043 Grenoble Cedex, France
}

\begin{abstract}
Third generation synchrotron light sources like the ESRF are characterised by a small momentum compaction in order to achieve a small horizontal emittance as well as by large sextupoles to correct the chromaticity and enlarge the dynamic aperture. Therefore the linear description of the optics is inadequate when considering the large excursions in momentum experienced by Touschek scattered particles. A good modelling of the non-linear behaviour is essential to better understand the limitations in the energy acceptance of the machine. Measurements of chromatic closed orbits, betatron tunes, and synchrotron frequency for momentum deviations up to $\pm 4 \%$ show a very strong non-linear behaviour on the negative energy side. A comparison is made of the experimental data with predictions from tracking simulations. Possible sources of disagreement are discussed.
\end{abstract}

\section{INTRODUCTION}

Lifetime is a key operational issue for third generation light sources, which are characterised by much smaller emittances and insertion device gaps than machines of the previous generation. Frequent refills due to a moderate lifetime lead to a reduction of the integrated brilliance. They induce a non-constant heat-load that implies changes in the thermal stability of beamline components, which in turn is detrimental to the quality of experiments.

Therefore achieving a large momentum acceptance is an essential feature in order to minimise the detrimental effects of Touschek scattering on the lifetime even for a high-energy ring like the $6 \mathrm{GeV}$ ESRF, where Touschek lifetime is an issue for time structure modes of operation (single bunch, 16 bunch). The study of the off-momentum particle behaviour is therefore of prime importance for understanding the limitations on the energy acceptance. This requires a good model of the machine.

The ESRF lattice is a Chasman-Green lattice with distributed dispersion providing a $4 \mathrm{~nm}$ horizontal emittance. Accordingly, a very small momentum compaction is achieved $\left(\alpha=1.8410^{-4}\right)$. In order to fight against the resistive wall and head-tail instabilities, natural chromaticities $\left(\Delta v_{x / z} /(\Delta \mathrm{p} / \mathrm{p})=-132 /-52\right)$ have to be corrected to positive values (from $+4.5 /+6$ in multibunch mode to $+7 /+13$ in single bunch mode) and strong harmonic sextupoles are used to enlarge the dynamic acceptance. These strong sextupoles introduce significant non-linearities in the lattice that affect off-momentum lattice characteristics (orbit, tunes, synchrotron frequency...). Higher order terms are required to provide an accurate description of the change of the orbit path length, the dispersion, the chromaticity or the synchrotron frequency. These expressions are expressed in the following equations:

$$
\begin{aligned}
& \frac{\Delta l}{l}=-\frac{\Delta f_{R F}}{f_{R F}}=\alpha_{1} \frac{\Delta p}{p}+\alpha_{2}\left(\frac{\Delta p}{p}\right)^{2}+\alpha_{3}\left(\frac{\Delta p}{p}\right)^{3}+\ldots \\
& \eta=\eta_{0}+\eta_{1} \frac{\Delta p}{p}+\eta_{2}\left(\frac{\Delta p}{p}\right)^{2}+\eta_{3}\left(\frac{\Delta p}{p}\right)^{3}+\ldots \\
& v=v_{0}+\xi_{1} \frac{\Delta p}{p}+\xi_{2}\left(\frac{\Delta p}{p}\right)^{2}+\xi_{3}\left(\frac{\Delta p}{p}\right)^{3}+\ldots \\
& f_{s}=f_{0}\left[\frac{h V_{R F} \cos \varphi_{s}}{2 \pi(E / e)}\right]^{\frac{1}{2}}\left[\alpha_{1}+2 \alpha_{2}\left(\frac{\Delta p}{p}\right)+3 \alpha_{3}\left(\frac{\Delta p}{p}\right)^{2}+\ldots\right]^{\frac{1}{2}}
\end{aligned}
$$

where $\alpha_{1}, \eta_{0}$ and $\xi_{1}$ are the first-order momentum compaction, dispersion and chromaticity, $f_{0}$ is the revolution frequency, $\mathrm{E}$ the machine energy, $\mathrm{h}$ the harmonic number, $V_{R F}$ the $R F$ voltage, $f_{R F}$ the $R F$ frequency and $\varphi_{\mathrm{s}}$ the synchronous phase

For a long time, experimental investigations of this non-linear behaviour and comparisons from tracking codes had shown significant discrepancies at large $\Delta \mathrm{p} / \mathrm{p}$ values. Several causes may account for this divergence: approximate model resulting from the sextupole description (calibration strength/current, thin/thick) and/or discarded multipolar components in magnets, validity of off-momentum tracking. After a careful analysis of these effects, a new sextupole model has been established which produces a good agreement with simulations in all off-momentum conditions.

\section{RELATION BETWEEN FREQUENCY AND ENERGY}

The usual experimental way of varying the momentum in the machine is by changing the RF frequency and using the definition of the momentum compaction $\alpha$ to deduce the off-momentum value from $\frac{\Delta f_{R F}}{f_{R F}}=-\alpha \frac{\Delta p}{p}$. According to Eq. (1), higher order terms in $\Delta \mathrm{p} / \mathrm{p}$ have to be included.

Tracking results have to be used to derive the experimental law. The orbit path length variation with momentum is computed for a finite number of $\Delta \mathrm{p} / \mathrm{p}$. Several tracking methods could be compared: BETA [1] at second order and symplectic tracking using thin lens and exact dipole tracking [2]. The results are in very good agreement. The experimental $\Delta \mathrm{f}_{\mathrm{RF}}$ values are then expressed in terms of $\Delta \mathrm{p} / \mathrm{p}$ values by applying a polynomial fit of degree 20 of the function $\Delta \mathrm{p} / \mathrm{p}=\mathrm{f}\left(\Delta \mathrm{f}_{\mathrm{RF}}\right)$. 
Figure 1 shows the strong deviation from the linear behaviour for positive $\Delta \mathrm{p} / \mathrm{p}$.

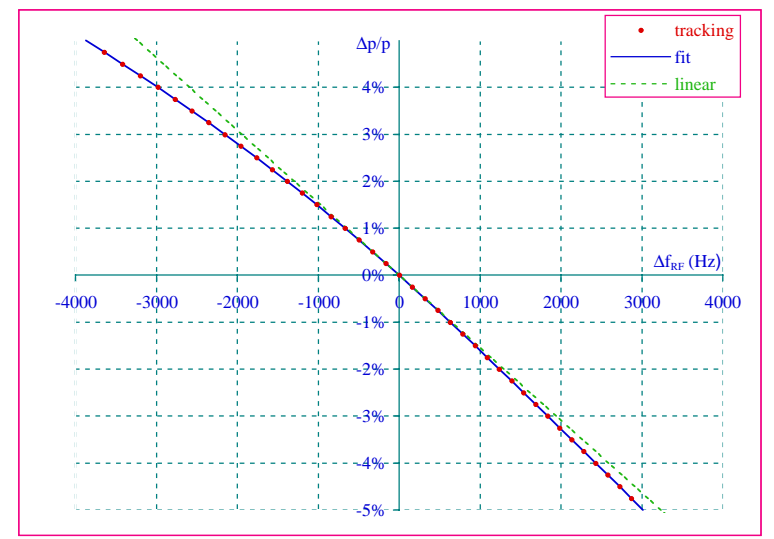

Figure 1: Frequency shift with energy

\section{EXPERIMENTAL OFF-MOMENTUM CHARACTERISTICS}

Off-momentum lattice characteristics have been measured for several sextupole settings by varying the RF frequency. Different features (large dynamic acceptance, minimum tune shifts with amplitude or enhanced contribution of the non-linear terms) characterise the sextupole settings but identical properties are obtained, namely an unsymmetrical behaviour for positive and negative $\Delta \mathrm{p} / \mathrm{p}$ deviations [3], [4].

- For the chromatic orbit (Figure 2), the main deviations between the measurements and the model appear for large orbit excursions, mainly on BPM 5 (middle of the achromat). The linearity of beam position measurements can explain part of the deviation.

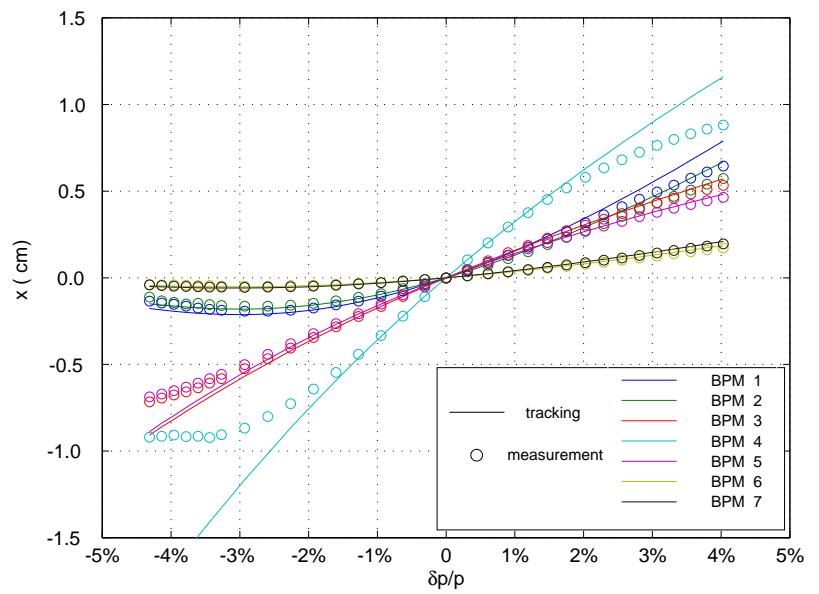

Figure 2: Chromatic closed orbits

- Large local chromaticities are observed in the horizontal plane for negative momentum deviations (Figure 3). Numerous synchrotron satellites can be observed on a tune spectrum analyser.

- Significant changes in the synchrotron frequency behaviour (Figure 4) occur for different sets of sextupoles tuned so as to vary the $\alpha_{2}$ term in a wide range.

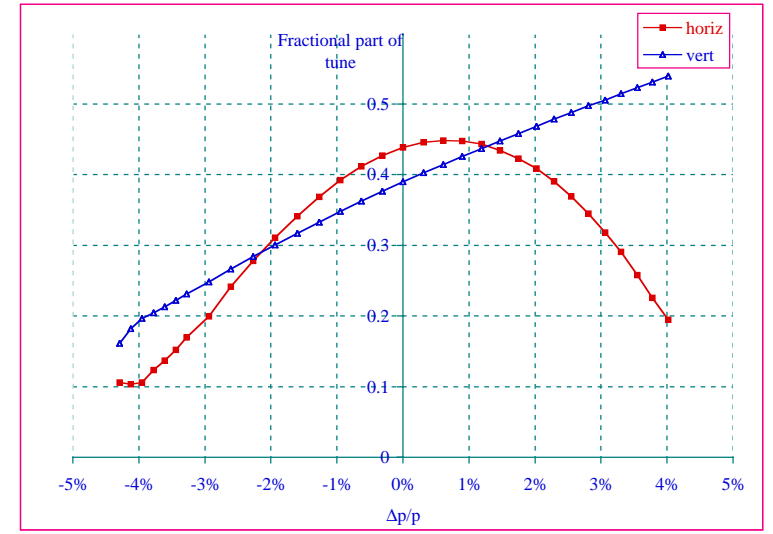

Figure 3: Tune shifts with energy

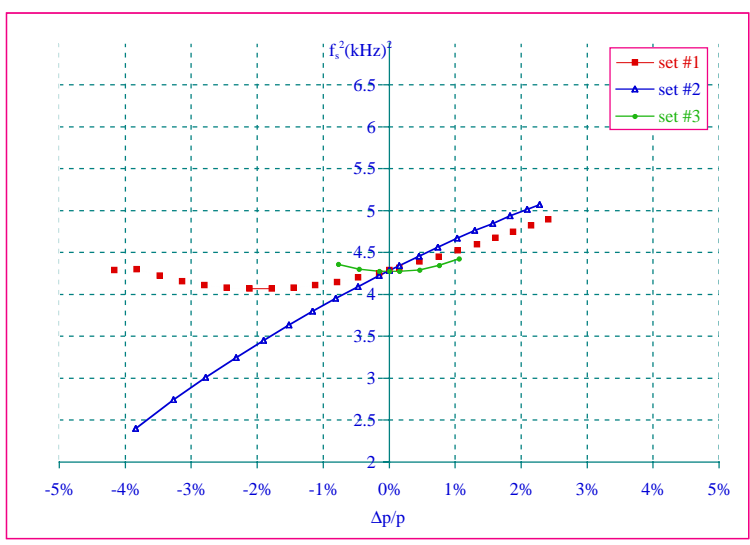

Figure 4: Synchrotron frequency evolution with energy

- Off-momentum horizontal $\beta$-functions (Figure 5) are processed from measurements of the centre of charge position of the beam recorded over a large number of turns by a turn-by-turn BPM system. The fast decoherence for large negative $\Delta \mathrm{p} / \mathrm{p}$ values makes the analysis rather inaccurate.

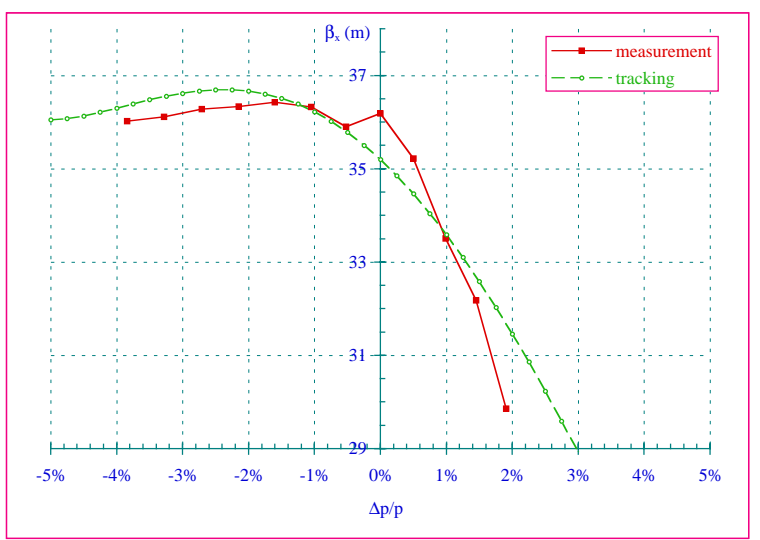

Figure 5: Horizontal $\beta$-functions

\section{SEXTUPOLE MODEL}

\subsection{Initial model}

Tracking simulations use sextupole strengths $H l=\frac{1}{2 B \rho} \frac{d^{2} B}{d x^{2}} l$ (B $\rho$ being the particle rigidity) and thin 
lens description with localised elements. In the real life, sextupoles are lumped magnets characterised by their current I.

The calibration of sextupoles yielding the relation $\mathrm{Hl}=\mathrm{f}$ (I) had been initially derived from magnetic measurements and chromaticity measurements with beam. A scan in current was applied to each sextupole family. Sextupole strengths that reproduce the measured chromaticities were fitted in a tracking code and compared to the magnetic measurement data.

Measurements of lattice off-momentum characteristics show a significant discrepancy with predictions from the model at large $\Delta \mathrm{p} / \mathrm{p}$. One typical example with dedicated sextupole settings enhancing the contribution of high order terms is shown in Figure 6. The measured tune paths significantly diverge from the model for $\Delta \mathrm{p} / \mathrm{p}> \pm 2$ $\%$. The divergence concerns mainly the horizontal tunes.

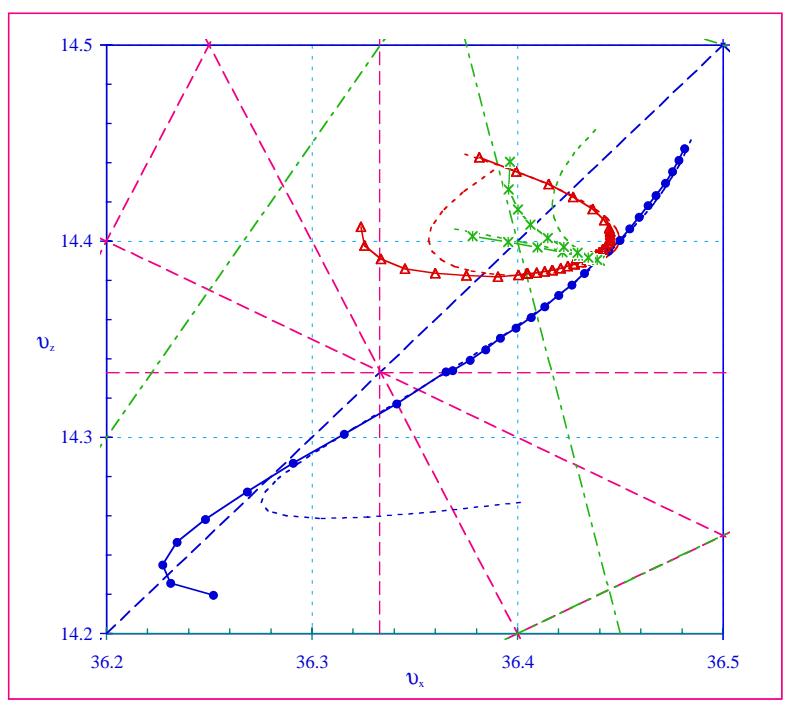

Figure 6: Measured and predicted (dashed) tune paths

\subsection{Tracking limitations}

Off-momentum tracking always implies some approximations in the description of elements (for instance the edge focusing in bending magnets) that may be responsible for the discrepancy between measurements and predictions. The comparison of BETA and MAD [5] results show that, at least in a $\pm 4 \% \Delta \mathrm{p} / \mathrm{p}$ range, the theoretical evolution of tunes with energy is very similar.

Non-linear effects due to higher order field components of magnetic elements could also explain the disagreement. The introduction of multipolar terms deduced from the results of magnetic measurements, as thin lens, in the tracking code yields no effect on the evolution of tunes with energy unless a significant scaling factor is applied. Even so, the disagreement with experimental results persists.

A noticeable change in the theoretical tune shifts with energy occurs when approximating the thick lens sextupoles by a succession of thin lenses as opposed to a single thin lens. This is the most significant effect.

\subsection{Refined model}

Starting from this revised description of the optics, the non-linear tune shifts with momentum measured over a wide range of $\Delta \mathrm{p} / \mathrm{p}$ values and for a large number of sextupole settings are used for fitting the sextupole strengths on the model. The method consists in launching a global fit on all sextupoles to minimise the differences between experimental and predicted $\Delta v=f(\Delta p / p)$ curves [6]. As illustrated in Figure 7 for horizontal tune shifts with energy and in Table 1 for the non-linear momentum compaction factor $\alpha_{2}$, a good agreement between measured and predicted characteristics is obtained.

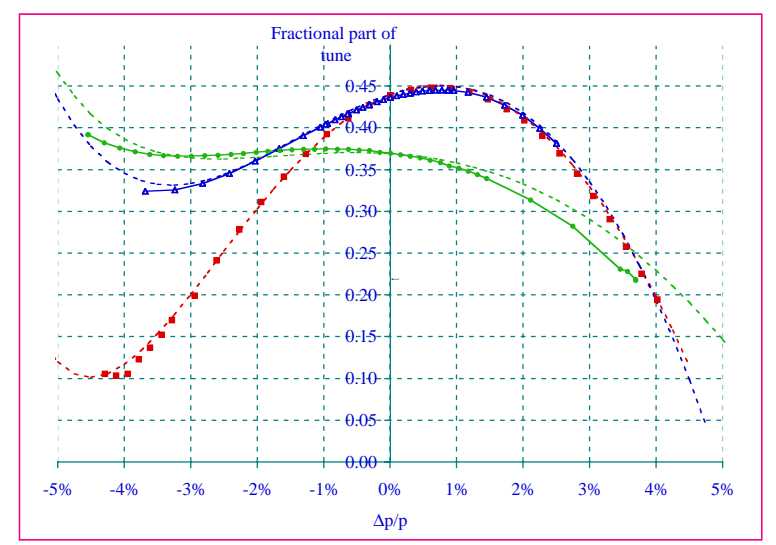

Figure 7: Comparison of measured (plain) and predicted (dashed) tune shifts with energy

Table 1: Comparison of $\alpha_{2}$ values

\begin{tabular}{|l|c|c|c|}
\hline & Measured & Analytical & Tracking \\
\hline Set \#1 & $4.210^{-4}$ & $5.610^{-4}$ & $5.810^{-4}$ \\
\hline Set \#2 & $7.810^{-4}$ & $1.010^{-3}$ & $1.010^{-3}$ \\
\hline Set \#3 & $-2.110^{-5}$ & $1.610^{-4}$ & $1.510^{-4}$ \\
\hline
\end{tabular}

\section{CONCLUSIONS}

The very non-linear off-momentum behaviour of the ESRF lattice is well reproduced in simulations. This gives confidence that the model may be used for pursuing the optimisation of the energy acceptance of the machine.

\section{REFERENCES}

[1] L. Farvacque, J.L. Laclare, A. Ropert, "BETA user's guide", ESRF-SR/LAT-88-08

[2] L. Michelotti, "Beamline user's guide: a C++ library for modelling beamlines and accelerators", 1995

[3] A. Ropert, "Non-linear behaviour of the low $\beta_{z}$ optics", ESRF 40-00/MDT

[4] L. Farvacque, "Measurement of the non-linear momentum compaction factor", ESRF 02-01/MDT

[5] F.C. Iselin, J.M. Jovett, J. Pancin, A. Adelmann, "MAD Version 9", EPAC2000, Vienna, 2000

[6] A. Ropert, "Recalibration of sextupoles via tune shifts with momentum measurements", ESRF 01-01/MDT 